\title{
High-electromechanical-coupling-coefficient and High-velocity Surface Acoustic Wave Materials of ZnO Films on Diamond
}

\author{
Jsung-Ta Tasi ${ }^{1}$ and Sean $\mathrm{Wu}^{2 *}$ \\ ${ }^{1}$ College of Robotics, Fuzhou Polytechnic, \\ No. 8 Lianrong Road, Fuzhou University Town, Fujian Province 350108, China \\ ${ }^{2}$ Department of Digital Game and Animation Design, Tung Fang Design University, \\ No. 110, Dongfang Rd., Hunei Dist., Kaohsiung City 82941, Taiwan
}

(Received July 13, 2018; accepted October 25, 2018)

Keywords: SAW, (100) ZnO, diamond

Functional coatings are effective for improving the properties of devices. The development of thin-film-based surface acoustic wave (SAW) devices coated with piezoelectric zinc oxide ( $\mathrm{ZnO}$ ) thin films has attracted considerable attention. The orientation and thickness of piezoelectric films will affect the characteristics of SAW devices. In this research, (100) $\mathrm{ZnO}$ films were combined with diamond to form new composite SAW substrates. The SAW properties of (100) $\mathrm{ZnO}$ films on diamond with four composite structures, namely, interdigital transducer (IDT)/(100)ZnO/diamond, (100)ZnO/IDT/diamond, IDT/(100)ZnO/metal/diamond, and metal/ (100) ZnO/IDT/diamond were systematically analyzed and some excellent SAW properties were observed. In particular, for mode 1 of (100)ZnO/IDT/diamond, a maximum $K^{2}$ of $5.85 \%$ and a maximum velocity of $8857 \mathrm{~m} / \mathrm{s}$ were obtained at a minimum film thickness ratio of $h / \lambda=0.11$. Such research results provide a predictable theoretical basis for the further application of such substrates in high-velocity SAW devices.

\section{Introduction}

Diamond is a non-piezoelectric material that requires a piezoelectric film to be deposited on top to excite surface acoustic waves (SAWs). A piezoelectric film on diamond is an attractive material for high-frequency SAW devices because the SAW velocity of diamond is the highest among all materials. Zinc oxide $(\mathrm{ZnO})$ films have been widely investigated for application in SAW and film bulk acoustic wave devices because of their high piezoelectric coupling factor. There are many researchers preparing (002) $\mathrm{ZnO}$ films on diamond to excite SAWs ${ }^{(1-10)}$ for high-frequency SAW devices. In particular, the commercialization of SAW devices based on the $(002) \mathrm{ZnO} /$ diamond structure has been developed by Sumitomo Electric Industries. ${ }^{(11,12)}$

Different orientations of piezoelectric films will form different acoustic properties. $\mathrm{ZnO}$ and AlN have the same crystalline structure. (100) AlN films on diamond exhibit excellent theoretical SAW properties compared with (002) AlN films on diamond. ${ }^{(13)}$ (100) ZnO films can be deposited by different methods. ${ }^{(14-18)}$ Bulk acoustic wave properties of (100) $\mathrm{ZnO}$

*Corresponding author: e-mail: wusean.tw@gmail.com

https://doi.org/10.18494/SAM.2019.2110 
films have been reported, ${ }^{(19)}$ but their SAW properties were seldom studied. In our previous research, ${ }^{(20)}$ for the first time, (100) $\mathrm{ZnO}$ films were combined with diamond to form new highfrequency SAW substrates. The five Rayleigh SAW modes of the composite structure [interdigital transducer (IDT)/(100)ZnO/diamond] exhibited excellent SAW properties. For a composite thinfilm SAW device, there are four basic structures, namely, IDT/(100)ZnO/diamond, (100)ZnO/ IDT/diamond, IDT/(100)ZnO/metal/diamond, and metal/(100)ZnO/IDT/diamond. Different composite structures will exhibit different SAW properties. In this research, the first five SAW modes propagating in the four composite structures have been systematically analyzed, and such structures have been determined in high-velocity SAW devices.

\section{Method of Analysis}

The layered piezoelectric structure, as depicted in Fig. 1, consists of a $\mathrm{ZnO}$ film ( $h$ : film thickness) and diamond. Figure 1 shows a SAW propagating on the $y-z$ plane for $(100) \mathrm{ZnO} /$ diamond. Following an approach similar to that developed by Campbell and Jones, ${ }^{(21)}$ the matrix method is effectively employed here to calculate the SAW velocity in a layered piezoelectric structure.

The acoustic and electric fields in mediums 1 and 2 can be expressed as

$$
\begin{gathered}
u_{j}^{(1)}=\sum_{m=1}^{4} C_{m} a_{j}^{(m)} \exp \left(i k b^{(m)} z\right) \exp [i k(P x-v t)], j=1,2,3, \\
\phi^{(1)}=\sum_{m=1}^{4} C_{m} a_{4}^{(m)} \exp \left(i k b^{(m)} z\right) \exp [i k(P x-v t)], \\
u_{j}^{(2)}=\sum_{m=1}^{8} X_{m} \alpha_{j}^{(m)} \exp \left(i k \beta^{(m)} z\right) \exp [i k(P x-v t)], \\
u_{j}^{(2)}=\sum_{m=1}^{8} X_{m} \alpha_{4}^{(m)} \exp \left(i k \beta^{(m)} z\right) \exp [i k(P x-v t)],
\end{gathered}
$$

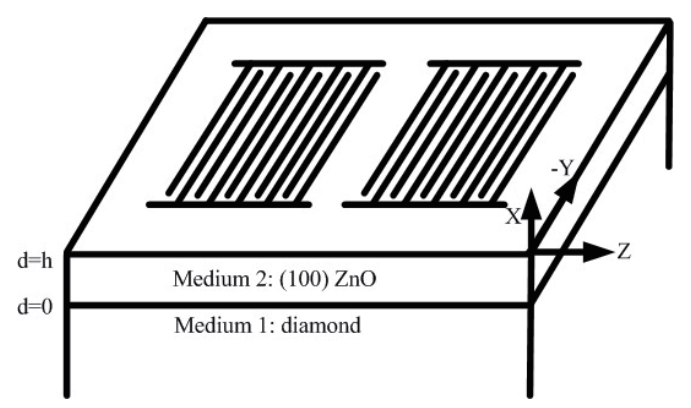

Fig. 1. Schematic of layered piezoelectric structure (100) $\mathrm{ZnO} /$ diamond. 
where $u$ is the acoustic displacement, $\phi$ the electric potential, $v$ the phase velocity, $k$ the wave number in the $x$-direction, and $P=1+i \gamma$, with $\gamma$ being the attenuation coefficient; moreover, $\beta$ is the wave number ratio, and $\alpha$ the associated partial field amplitude. Substituting Eqs. (1) and (2) into stiffened Christoffel equations yields an eight-order algebraic equation for the wave number ratio $b$. Thus, for each pair of $(v, \gamma)$ values, there are eight real or complex $b$ values. For a semi-infinite piezoelectric crystal, i.e., medium 1 in this structure, four complex roots with negative imaginary parts are selected for the Rayleigh type. In medium 2 , all eight roots of $\beta$ are selected.

The boundary conditions require that the acoustic displacements and stresses be continuous at $d=0$, and that the stress-free surface be assumed at $d=h$. In addition, the electric potential and normal component of electric displacement must be continuous at the interface for an electrically free surface. For a metalized (thin metal film) surface, electric potential is not observed. By substituting Eqs. (1)-(4) into the boundary conditions, the phase velocity $v$ and the attenuation coefficient $\gamma$ can be obtained numerically. The electromechanical coupling coefficient $\left(K^{2}\right)$ can be calculated from

$$
K^{2}=2 \frac{v_{f}-v_{m}}{v_{f}}
$$

where $v_{f}$ and $v_{m}$ are the phase velocities obtained when the electrical boundary conditions at the interface at which the IDT is placed are assumed to be electrically free and shorted, respectively. The material constants of $\mathrm{ZnO}$ and diamond employed here were obtained from the study reported by Gualtieri et al. ${ }^{(22)}$ and Benetti et al. ${ }^{(23)}$

\section{Results and Discussion}

The phase velocity dispersion curves of the first five SAW modes propagating in the IDT/ $\mathrm{ZnO} /$ diamond structure are shown in Fig. 2. The curves are plotted as functions of the film

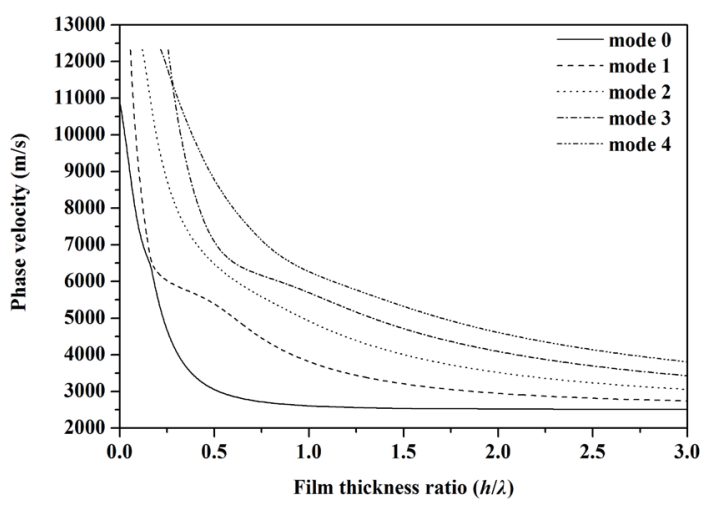

Fig. 2. Calculated phase velocity dispersion curves of first five SAW modes propagating in the (100) $\mathrm{ZnO} /$ diamond structure. 
thickness ratio $(h / \lambda)$, where $h$ is the $\mathrm{ZnO}$ film thickness and $\lambda$ is the wavelength. The phase velocity of each mode decreases as the film thickness ratio increases. For mode 0, the phase velocity originates from the SAW velocity of diamond $(10934 \mathrm{~m} / \mathrm{s})$ at $h / \lambda=0$. As the film thickness ratio $(h / \lambda)$ increases, the phase velocity decreases. At $h / \lambda=3$, the velocity of mode 0 is close to the SAW velocity of (100) $\mathrm{ZnO}(2508 \mathrm{~m} / \mathrm{s})$. Modes $1,2,3$, and 4 show the cutoff at the critical point, where the phase velocity is close to the shear bulk wave velocity in diamond $(12323 \mathrm{~m} / \mathrm{s})$. Note that the phase velocity dispersion curve of mode 0 is very close to that of mode 1 , and that the phase velocity dispersion curve of mode 3 is very close to that of mode 4 , but do not cross. Instead of decreasing gradually, mode 0 bends up and mode 1 bends down at $h / \lambda=0.17$, and mode 3 bends up and mode 4 bends down at $h / \lambda=0.28$. This phenomenon was also observed in the (002) $\mathrm{ZnO}$ /diamond structure, as mentioned in some earlier papers. ${ }^{(24,25)}$

The $K^{2}$ dispersion curves of the first five Rayleigh-type modes propagating in the four composite structures are shown in Figs. 3-6. The $K^{2}$ dispersion curves are calculated for the four composite structures as described below.

\subsection{Electromechanical coupling coefficient dispersion curves of IDT/ZnO/diamond structure}

The $K^{2}$ dispersion curves of the IDT/(100)ZnO/diamond structure are shown in Fig. 3. For mode 0 , the $K^{2}$ curve shows an optimal value $(1.57 \%)$ at $h / \lambda=0.15$ and the velocity is $6670 \mathrm{~m} / \mathrm{s}$. For mode $1, K^{2}$ increases from $0.05 \%$ at $h / \lambda=0.06$. The $K^{2}$ curve shows an optimal value (4.28\%) at $h / \lambda=0.42$ and the velocity is $5616 \mathrm{~m} / \mathrm{s}$. For mode $2, K^{2}$ increases from $0.06 \%$ at $h / \lambda=0.12$. The $K^{2}$ curve shows an optimal value $(1.67 \%)$ at $h / \lambda=0.81$ and the velocity is $5409 \mathrm{~m} / \mathrm{s}$. For mode 3 , $K^{2}$ increases from $0.002 \%$ at $h / \lambda=0.22$. The $K^{2}$ curve shows an optimal value $(1.7 \%)$ at $h / \lambda=0.99$ and the velocity is $5714 \mathrm{~m} / \mathrm{s}$. For mode $4, K^{2}$ increases from $0.2 \%$ at $h / \lambda=0.26$. The $K^{2}$ curve shows an optimal value $(1.34 \%)$ at $h / \lambda=1.32$ and the velocity is $5641 \mathrm{~m} / \mathrm{s}$.

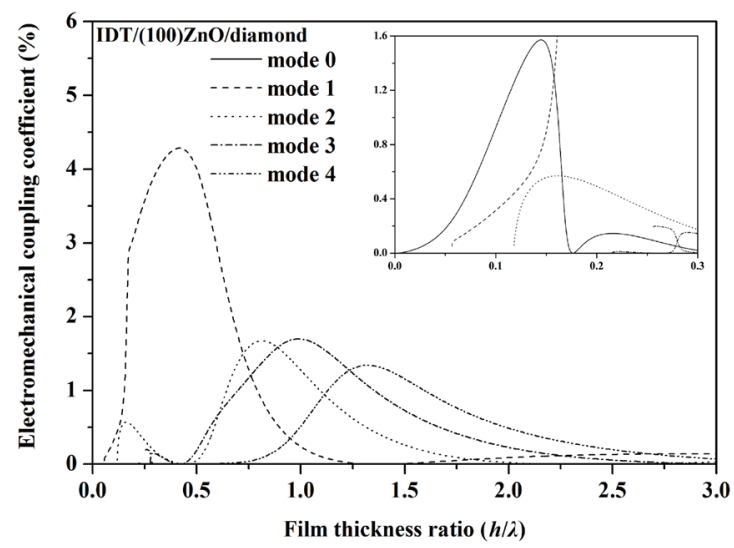

Fig. 3. Calculated $K^{2}$ dispersion curves of first five Rayleigh SAW modes propagating in IDT/(100)ZnO/diamond. 


\subsection{Electromechanical coupling coefficient dispersion curves of $\mathrm{ZnO} / \mathrm{IDT} / \mathrm{diamond}$ structure}

The $K^{2}$ dispersion curves of the (100)ZnO/IDT/diamond structure are shown in Fig. 4. For mode 0 , the $K^{2}$ curve shows an optimal value $(3.25 \%)$ at $h / \lambda=0.35$ and the velocity is $3676 \mathrm{~m} / \mathrm{s}$. For mode $1, K^{2}$ increases from $1.03 \%$ at $h / \lambda=0.06$. The $K^{2}$ curve shows an optimal value $(5.85 \%)$ at $h / \lambda=0.11$ and the velocity is $8587 \mathrm{~m} / \mathrm{s}$. For mode $2, K^{2}$ increases from $0.04 \%$ at $h / \lambda=0.12$. The $K^{2}$ curve shows an optimal value $(0.69 \%)$ at $h / \lambda=1.59$ and the velocity is $3894 \mathrm{~m} / \mathrm{s}$. For mode $3, K^{2}$ increases from $0.002 \%$ at $h / \lambda=0.22$. The $K^{2}$ curve shows an optimal value $(1.88 \%)$ at $h / \lambda=0.55$ and the velocity is $6748 \mathrm{~m} / \mathrm{s}$. For mode $4, K^{2}$ increases from $0.15 \%$ at $h / \lambda=0.26$. The $K^{2}$ curve shows an optimal value $(0.83 \%)$ at $h / \lambda=0.85$ and the velocity is $6694 \mathrm{~m} / \mathrm{s}$.

\subsection{Electromechanical coupling coefficient dispersion curves of IDT/ZnO/metal/diamond structure}

Figure 5 shows the $K^{2}$ dispersion curves of the IDT/(100)ZnO/metal/diamond structure. For mode 0 , the $K^{2}$ curve shows an optimal value $(1.33 \%)$ at $h / \lambda=1.17$ and the velocity is $2564 \mathrm{~m} / \mathrm{s}$. For mode $1, K^{2}$ increases from $1.32 \%$ at $h / \lambda=0.06$. The $K^{2}$ curve shows the optimal value $(4.15 \%)$ at $h / \lambda=0.45$ and the velocity is $5529 \mathrm{~m} / \mathrm{s}$. For mode $2, K^{2}$ increases from $0.06 \%$ at $h / \lambda=0.12$. The $K^{2}$ curve shows an optimal value $(1.7 \%)$ at $h / \lambda=0.82$ and the velocity is $5389 \mathrm{~m} / \mathrm{s}$. For mode 3 , $K^{2}$ increases from $0.001 \%$ at $h / \lambda=0.22$. The $K^{2}$ curve shows an optimal value $(1.69 \%)$ at $h / \lambda=1$ and the velocity is $5703 \mathrm{~m} / \mathrm{s}$. For mode $4, K^{2}$ increases from $0.33 \%$ at $h / \lambda=0.26$. The $K^{2}$ curve shows an optimal value (1.34\%) at $h / \lambda=1.33$ and the velocity is $5623 \mathrm{~m} / \mathrm{s}$.

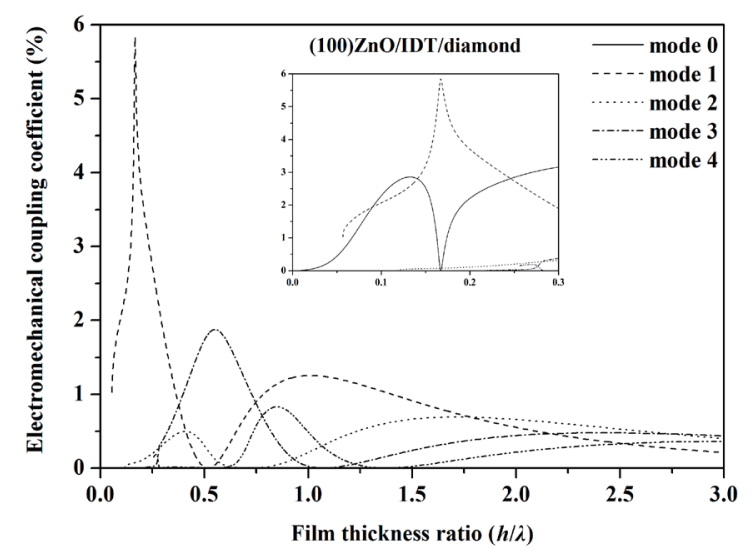

Fig. 4. Calculated $K^{2}$ dispersion curves of first five Rayleigh SAW modes propagating in (100)ZnO/IDT/ diamond.

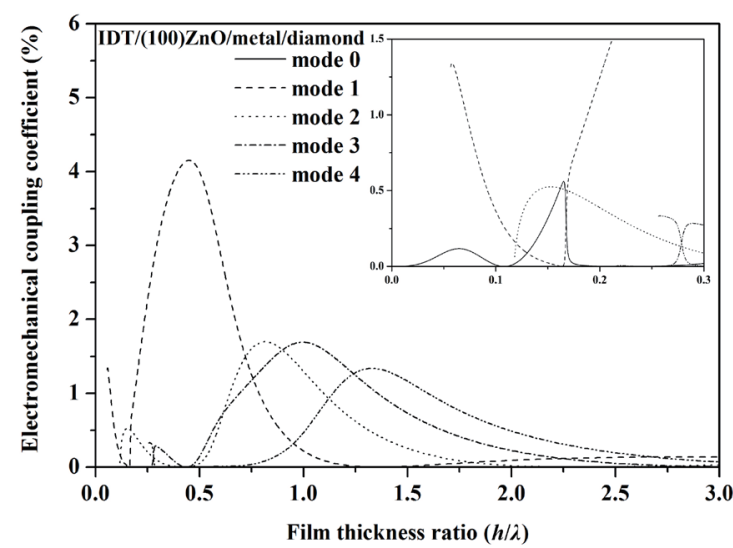

Fig. 5. Calculated $K^{2}$ dispersion curves of first five Rayleigh SAW modes propagating in IDT/(100)ZnO/ metal/diamond. 


\subsection{Electromechanical coupling coefficient dispersion curves of metal/ZnO/IDT/diamond structure}

Figure 6 shows the $K^{2}$ dispersion curves of the metal/(100)ZnO/IDT/diamond structure. For mode 0 , the $K^{2}$ curve shows an optimal value (3.33\%) at $h / \lambda=0.36$ and the velocity is $3551 \mathrm{~m} / \mathrm{s}$. For mode $1, K^{2}$ increases from $2.29 \%$ at $h / \lambda=0.06$. The $K^{2}$ curve shows an optimal value $(3.73 \%)$ at $h / \lambda=0.17$ and the velocity is $6338 \mathrm{~m} / \mathrm{s}$. For mode $2, K^{2}$ increases from $0.01 \%$ at $h / \lambda=0.12$. The $K^{2}$ curve shows an optimal value $(0.69 \%)$ at $h / \lambda=1.7$ and the velocity is $3759 \mathrm{~m} / \mathrm{s}$. For mode 3 , $K^{2}$ increases from 0 at $h / \lambda=0.22$. The $K^{2}$ curve shows an optimal value $(1.86 \%)$ at $h / \lambda=0.56$ and the velocity is $6618 \mathrm{~m} / \mathrm{s}$. For mode $4, K^{2}$ increases from $0.29 \%$ at $h / \lambda=0.26$. The $K^{2}$ curve shows an optimal value $(0.82 \%)$ at $h / \lambda=0.85$ and the velocity is $6676 \mathrm{~m} / \mathrm{s}$.

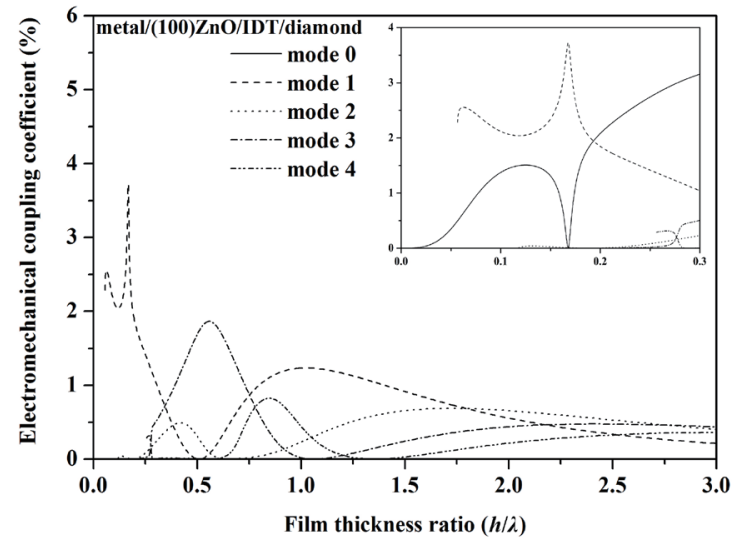

Fig. 6. Calculated $K^{2}$ dispersion curves of first five Rayleigh SAW modes propagating in metal/(100)ZnO/IDT/ diamond.

Table 1

Relative maximum $K^{2}$ values of the first five modes propagating in the four structures.

\begin{tabular}{|c|c|c|c|c|}
\hline Propagation mode & Structure & $h / \lambda$ & $K^{2}(\%)$ & Velocity $(\mathrm{m} / \mathrm{s})$ \\
\hline \multirow{4}{*}{ Mode 0} & IDT/(100)ZnO/diamond & 0.15 & 1.57 & 6670 \\
\hline & (100) $\mathrm{ZnO} / \mathrm{IDT} /$ diamond & 0.35 & 3.25 & 3676 \\
\hline & IDT/(100) $\mathrm{ZnO} /$ metal/diamond & 1.17 & 1.33 & 2564 \\
\hline & metal/(100)ZnO/IDT/diamond & 0.36 & 3.33 & 3551 \\
\hline \multirow{4}{*}{ Mode 1} & IDT/(100)ZnO/diamond & 0.42 & 4.28 & 5616 \\
\hline & (100)ZnO/IDT/diamond & 0.11 & 5.85 & 8587 \\
\hline & $\mathrm{IDT} /(100) \mathrm{ZnO} / \mathrm{metal} /$ diamond & 0.45 & 4.15 & 5529 \\
\hline & metal/(100)ZnO/IDT/diamond & 0.17 & 3.73 & 6338 \\
\hline \multirow{4}{*}{ Mode 2} & IDT/(100)ZnO/diamond & 0.81 & 1.67 & 5409 \\
\hline & $(100) \mathrm{ZnO} / \mathrm{IDT} /$ diamond & 1.59 & 0.69 & 3894 \\
\hline & IDT/(100) $\mathrm{ZnO} / \mathrm{metal} /$ diamond & 0.82 & 1.7 & 5389 \\
\hline & metal/(100)ZnO/IDT/diamond & 1.7 & 0.69 & 3759 \\
\hline \multirow{4}{*}{ Mode 3} & IDT/(100)ZnO/diamond & 0.99 & 1.7 & 5714 \\
\hline & (100)ZnO/IDT/diamond & 0.55 & 1.88 & 6748 \\
\hline & IDT/(100) ZnO/metal/diamond & 1 & 1.69 & 5703 \\
\hline & metal/(100)ZnO/IDT/diamond & 0.56 & 1.86 & 6618 \\
\hline \multirow{4}{*}{ Mode 4} & IDT/(100)ZnO/diamond & 1.32 & 1.34 & 5641 \\
\hline & (100)ZnO/IDT/diamond & 0.85 & 0.83 & 6694 \\
\hline & IDT/(100) $\mathrm{ZnO} /$ metal/diamond & 1.33 & 1.34 & 5623 \\
\hline & $\mathrm{metal} /(100) \mathrm{ZnO} / \mathrm{IDT} /$ diamond & 0.85 & 0.82 & 6676 \\
\hline
\end{tabular}


Note that the $K^{2}$ dispersion curves change markedly at $h / \lambda=0.17$ and 0.28 , as shown in the insets in Figs. 3-6. In accordance with the rapid change in phase velocity, the $K^{2}$ dispersion curves change markedly. The $K^{2}$ dispersion curve of mode 0 decreases and the $K^{2}$ dispersion curve of mode 1 increases markedly at $h / \lambda=0.17$. The $K^{2}$ dispersion curve of mode 3 increases and the $K^{2}$ dispersion curve of mode 4 decreases markedly at $h / \lambda=0.28$. For the first five modes of the four structures, the relative maximum $K^{2}$ values are summarized in Table 1 . It is obvious that mode 1 of the (100) ZnO/IDT/diamond structure has a maximum $K^{2}$ of $5.85 \%$ and a maximum velocity of $8857 \mathrm{~m} / \mathrm{s}$ at a minimum film thickness ratio of $h / \lambda=0.11$.

\section{Conclusions}

In this research, (100) $\mathrm{ZnO}$ films were combined with diamond to form new composite SAW substrates. The SAW properties of (100) $\mathrm{ZnO}$ films on diamond were systematically analyzed for four composite structures, namely, IDT/(100)ZnO/diamond, (100)ZnO/IDT/diamond, IDT/ (100) $\mathrm{ZnO} / \mathrm{metal} /$ diamond, and metal/(100)ZnO/IDT/diamond with excellent SAW properties. In particular, for mode 1 of (100)ZnO/IDT/diamond, a maximum $K^{2}$ of $5.85 \%$ and a maximum velocity of $8857 \mathrm{~m} / \mathrm{s}$ were obtained at a minimum film thickness ratio of $h / \lambda=0.11$. These research results provide a predictable theoretical basis for the further application of such substrates in high-velocity SAW devices.

\section{Acknowledgments}

We thank Fuzhou Polytechnics for financial support (project code: RCQD201703).

\section{References}

1 M. Davydova, A. Laposa, J. Smarhak, A. Kromka, N. Neykova, J. Nahlik, J. Kroutil, J. Drahokoupil, and J. Voves: Beilstein J. Nanotechnol. 9 (2018) 22. https://doi.org/10.3762/bjnano.9.4

2 S. I. Burkov, O. P. Zolotova, B. P. Sorokin, P. P. Turchin, and V. S. Talismanov: J. Acoust. Soc. Am. 143 (2018) 16. https://doi.org/10.1121/1.5019475

3 Y. Li, H. Li, M. Li, C. Li, Y. Lei, D. Sun, and B. Yang: J. Alloys Compd. 743 (2018) 187. https://doi.org/10.1016/ j.jallcom.2018.02.004

4 D. Kathiravan, B. R. Huang, A. Saravanan, C. J. Yeh, K. C. Leou, and I. N. Lin: Appl. Surf. Sci. 426 (2017) 630. https://doi.org/10.1016/j.apsusc.2017.07.194

5 Y. Q. Fu, L. Garcia-Gancedo, H. F. Pang, S. Porro, Y. W. Gu, J. K. Luo, X. T. Zu, F. Placido, J. I. B. Wilson, A. J. Flewitt, and W. I. Milne: Biomicrofluidics 6 (2012) 024105. https://doi.org/10.1063/1.3699974

6 H. F. Pang, L. G. Gancedo, Y. Q. Fu, S. Porro, Y. W. Gu, J. K. Luo, X. T. Zu, F. Placido, J. I. B. Wilson, A. J. Flewitt, and W. I. Milne: Phys. Status Solidi, A 210 (2013) 1575. https://doi.org/10.1002/pssa.201228631

7 A. Hachigo, H. Nakahata, K. Itakura, S. Fujii, and S. Shikata: IEEE Ultrason. Symp. 1 (1999) 325. https://doi. org/10.1109/ULTSYM.1999.849412

8 K. Higaki, H. Nakahata, H. Kitabayashi, S. Fujii, K. Tanabe, Y. Seki, and S. I. Shikata: IEEE Trans. Ultrason. Ferroelectr. Freq. Control 44 (1997) 1395. https://doi.org/10.1109/58.656643

9 T. Lamara, M. Belmahi, O. Elmazria, L. Le Brizoual, J. Bougdira, M. Re'my, and P. Alnot: Diam. Relat. Mater. 13 (2004) 581. https://doi.org/10.1016/j.diamond.2003.10.075

10 H. Nakahata, K. Higaki, A. Hachigo, S. Shikata, N. Fujimori, Y. Takahashi, T. Kajihara, and Y. Yamamoto: Jpn. J. Appl. Phys. 33 (1994) 324. http://iopscience.iop.org/article/10.1143/JJAP.33.324/meta

11 K. Itakura, A. Hachigo, H. Nakahata, S. Fujii, and S. Shikata: U.S. Patent No. 6469416 (2002). https://patents. google.com/patent/US6469416B1/en

12 K. Itakura, A. Hachigo, S. Fujii, H. Nakahata, and S. Shikata: U.S. Patent No. 6642813 (2003). https://patents. google.com/patent/US6642813B1/en 
13 S. Wu, R. Ro, Z. X. Lin, and M. S. Lee: J. Appl. Phys. 104 (2008) 064919. https://doi.org/10.1063/1.2986215

14 L. C. Chao, H. T. Hu, S. H. Yang, and Y. C. Fan: Thin Solid Films 516 (2008) 6305. https://doi.org/10.1016/ j.tsf.2007.12.091

15 M. Pacio, H. Juárez, G. Escalante, G. García, T. Díaz, and E. Rosendo: Mater. Sci. Eng., B 174 (2010) 38. https://doi.org/10.1016/j.mseb.2010.04.030

16 N. Fujimura, T. Nishihara, S. Goto, J. Xu, and T. 1to: J. Cryst. Growth 130 (1993) 269. https://doi. org/10.1016/0022-0248(93)90861-P

17 L. C. Nistor, C. Ghica, D. Matei, G. Dinescu, M. Dinescu, and G. Van Tendeloo: J. Cryst. Growth 277 (2005) 26. https://doi.org/10.1016/j.jcrysgro.2004.12.162

18 Y. Cui, G. Du, Y. Zhang, H. Zhu, and B. Zhang: J. Cryst. Growth 282 (2005) 389. https://doi.org/10.1016/ j.jcrysgro.2005.05.028

19 S. Wu, Z. X. Lin, M. S. Lee, and R. Ro: J. Appl. Phys. 102 (2007) 084908. https://doi.org/10.1063/1.2801403

20 S. Wu, R. Ro, and Z. X. Lin: Appl. Phys. Lett. 94 (2009) 032908. https://doi.org/10.1063/1.3074370

21 J. J. Campbell and W. R. Jones: IEEE Trans. Sonics and Ultrason. 15 (1968) 209. https://doi.org/10.1109/ T-SU.1968.29477

22 J. G. Gualtieri, J. A. Kosinski, and A. Ballato: IEEE Trans. Ultrason. Ferroelectr. Freq. Control 41 (1994) 53. https://doi.org/10.1109/58.265820

23 M. Benetti, D. Cannata, F. D. Pietrantonio, and E. Verona: IEEE Trans. Ultrason. Ferroelectr. Freq. Control 52 (2005) 1806. https://doi.org/10.1109/TUFFC.2005.1561635

24 E. L. Adler and L. Solie: IEEE Ultrason. Symp. 1 (1995) 341. https://doi.org/10.1109/ULTSYM.1995.495594

25 T. T. Wu and Y. Y. Chen: IEEE Trans. Ultrason. Ferroelectr. Freq. Control 49 (2002) 142. https://doi.org/ $10.1109 / 58.981392$

\section{About the Authors}

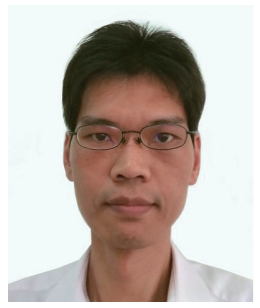

Jsung-Ta Tasi received his B.S., M.S., and Ph.D. degrees from National Cheng Kung University, Taiwan, in 1994, 1995, and 2000, respectively. From 2001 to 2007, he was an engineer in Darfon Electronics Corp., Taiwan. From 2007 to 2017, he was with the Department of Network and Communication, Taiwan ShouFu University as an assistant professor. Since 2017, he has been an associate professor in the College of Robotics, Fuzhou Polytechnic, China. His research interests are microwave dielectric materials, filters for RF communication, antenna switch modules of handset systems, and battery management systems. (tsaidadar@qq.com)

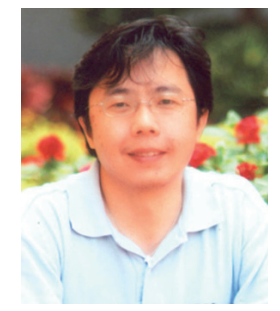

Sean Wu was born in Kaohsiung, Taiwan, on December 30, $1968 . \mathrm{He}$ received his B.S. degree in electrical engineering in 1993 from Chung Yuan Christian University in Taiwan, and his M.S. and Ph.D. degrees in electrical engineering from National Cheng Kung University in 1995 and 2001, respectively. He joined the Department of Electronics Engineering and Computer Science, Tung Fang Design University and became a professor in 2010. He also served as the Department Head of Electronics Engineering and Computer Science from 2006 to 2008. He was the Dean of Academic Affairs of Tung Fang Design University from 2011 to 2013. He was the recipient of the 2012 special talent excellent elasticity professor salary of the Taiwan Ministry of Education annual awards. His research interests are in the fabrication of piezoelectric thin films, design of acoustic wave devices, and substrate materials for SAW and FBAR devices. He has authored or coauthored over 100 technical journal and conference papers.

(wusean.tw@gmail.com) 